\title{
Nuclear energy and its ecological byproducts: Lessons from Chernobyl and Fukushima
}

\author{
Timothy A. Mousseau and Anders P. Møller
}

\section{Abstract}

Given increasing energy needs related to global development, and the spectre of climate change related to carbon dioxide $\left(\mathrm{CO}_{2}\right)$ emissions from fossil fuels, there is an urgent need for large-scale energy production that does not involve the production of greenhouse gases. Nuclear energy is one possible solution that has been embraced by developing and developed countries alike (for example, China and the US). But the accidents at Three Mile Island, Chernobyl, and most recently Fukushima have demonstrated the vulnerability of this technology to human error, design flaws, and natural disasters, and these accidents have resulted in enormous health, environmental, and economic costs that must be factored into any energy policy that includes nuclear as an option. In the past, such analyses have largely ignored the potential costs of accidents for ecological systems in affected regions. Studies of natural systems are essential since they provide a bellwether for the potential longterm consequences for human populations that by necessity and government policy continue to inhabit contaminated regions. In this chapter, we discuss studies of the non-human biota living in Chernobyl and Fukushima. Extensive research on birds, insects, rodents, microbes, and trees has demonstrated potentially significant injury to individuals, species, and ecosystem functioning related to radiation exposure that has previously been underappreciated. We present an overview of the effects of radiation on DNA, birth defects, infertility, cancer, and longevity, and its consequences for the health and long-term prospects of wildlife living in radioactive regions of the world. 


\section{Introduction}

The nuclear disasters at Chernobyl and Fukushima have had enormous direct economic impacts, estimated in the hundreds of billions of US dollars (Samet and Seo 2016), with much of these costs associated with the decommissioning of damaged reactors and clean-up of affected regions. And yet very little investment has been made into the ecological consequences of the radionuclides that were dispersed at continental scales. In large part, the lack of investment in basic scientific research has stemmed from the perception, often perpetuated by nuclear regulatory bodies, that the direct effects of these contaminants have been minimal. There have even been suggestions that the wildlife are thriving as a result of these disasters because of reduced hunting pressures in the exclusion zones, leaving the public with the notion that radioactive contaminants are of little concern. In this chapter, we review some of the recent scientific studies that have been conducted over the past decade aimed at assessing the health and population success of wildlife in Chernobyl and Fukushima in relation to radioactivity in these regions. It is proposed that studies of these accident sites can provide valuable insights to possible consequences for biota, and perhaps even humans, exposed to radioactivity from accidents and other sources.

\section{The hazards related to nuclear energy extend far beyond catastrophic accidents}

No matter how one personally feels about nuclear energy, the truth is that it is here now and will continue to be a significant component of the world's energy portfolio for many years to come. There are currently on the order of 438 nuclear reactors in operation around the world, and 65 more are under construction (for example, 22 in China and four in the US), with 165 more on order or planned, and 325 additional reactors proposed. Most people do not realise that every nuclear reactor generates large amounts of radioactive effluents as a normal part of day-to-day operations, although total emissions have dropped significantly over the past decades. For example, a typical boiling water reactor in the US generates between 1,000 and 100,000 gigabecquerels of radioactive noble gases each year, while pressurised water reactors generate 10 to 100 times less, on average (Burris et al. 2012). In addition, a potpourri of other, potentially more concerning radionuclides (for example, iodine-131, 
cesium-137, and strontium-90) are also produced and released by normal operations at nuclear power stations. Although there have been a few studies of the possible effects of such releases for humans (for example, childhood leukaemia; Fairlie 2014), studies of non-human biota have largely been limited to the effects of thermal pollution rather than any effects of radioactivity per se. Given the prevalence of nuclear power for the foreseeable future, basic studies of their impacts on ecological systems seem warranted.

In addition to lawful, regulated emissions from nuclear power plants, it is now evident that many of these facilities are leaking unregulated quantities of radioactive effluents into the environment. A notable recent discovery of this sort was at the Vermont Yankee nuclear power plant in the US, where in 2010 large unregulated leaks of tritium (radioactive hydrogen) were discovered and linked to faulty cooling pipes. The discoveries of these leaks undoubtedly played a significant role in the closure of this nuclear power plant in 2014. Since then, it has been reported that more than three-quarters of the US's commercial power plant sites have had some kind of radioactive leak. In part, such leaks are the inevitable consequence of an ageing nuclear fleet and it seems likely that many more leaks will be discovered in the future. Despite these obvious issues, very little is known about the potential ecological impacts of such emissions. A report commissioned by the US Government Accountability Office indicated that little was known about the hazards related to tritium leaks and that further research was warranted (US GAO 2011).

In fact, all parts of the nuclear fuel cycle release vast quantities of radioactive contaminants, from the tailings generated by mining operations, to the processing and packaging of nuclear fuels. At the end of the cycle, enormous spent fuel stockpiles have amassed at nuclear power stations over the course of their operations. All of these sources represent potential hazards to the surrounding human population and ecological landscape, especially in the event of accidents or natural disasters. However, 'events' at nuclear facilities are not well-documented and there have been few studies of their ecological consequences.

The potential risks associated with nuclear energy have recently been comprehensively explored by Wheatley, Sovacool, and Sornette (2016), where they estimated a 50 per cent chance of a Fukushima-scale event or larger in the next 50 years, a Chernobyl event (or larger) in the next 27 years, and a Three Mile Island event (or larger) in the next 10 years. 
Based on this analysis, future accidents appear inevitable, and yet there is still relatively little investment in the basic research needed to accurately assess the likely hazards to ecological systems from such accidents.

\section{A research program to assess ecological consequences of nuclear accidents}

In an attempt to at least partially fill the void in current knowledge concerning the hazards of radioactive contamination for natural systems, Timothy Mousseau and Anders Møller initiated a collaboration in 2000 to investigate the consequences of the Chernobyl disaster on bird populations in the region. In 2005 , these studies expanded to include a variety of organisms, including insects, spiders, and plants, and research in Fukushima began in July 2011. The organising principles used to direct this research initiative were related to the following questions:

1. Do the radiation levels observed in Chernobyl (and now Fukushima) generate doses sufficient to increase mutation rates and genetic damage in natural populations?

2. Are there phenotypic consequences to elevated mutation rates and genetic damage in these regions?

3. Are there fitness consequences (i.e. changes in survival and/or reproduction) of elevated mutation rates?

4. Is there any evidence that populations are adapting to elevated radiation levels in these regions?

5. Are there consequences of radiation effects for abundance and diversity of natural populations?

6. Are there ecosystem effects that result from radiation effects on populations?

\section{Genetic effects of radiation on non-human biota}

Often, the first thought that comes to mind when discussing radioactivity concerns the possible genetic consequences of exposure to this mutagen. There is now an overwhelming body of evidence to suggest that, indeed, genetic systems are directly affected by chronic exposure to low doses 
of ionising radiation in the environment. The evidence comes from a plethora of single species studies, and more recently from meta-analyses of compilations of these single species studies.

Perhaps the first test for radiation effects on nuclear DNA mutation rates for a Chernobyl population used microsatellite markers (i.e. DNA fingerprints) to examine de novo mutation rates in barn swallows (Hirundo rustica) by comparing microsatellite DNA fingerprints for parents and their offspring (reviewed in Mousseau and Møller 2014). This study found mutation rates for these markers to be two- to 10 -fold higher in Chernobyl when compared to control populations in Ukraine and Italy, a finding that was paralleled by a study of the offspring of Chernobyl accident liquidators (i.e. humans). Surprisingly, there have been no other similar studies to assess de novo, heritable genetic mutations related to the Chernobyl accident. Given the plummeting costs of the genomic tools needed to assess changes at the level of individual DNA, much progress could be made towards a fundamental understanding of how the interaction between mutagens and genes are induced and transmitted to subsequent generations. Such studies are greatly needed, not just for issues related to conservation biology, but also to address fundamental questions in evolutionary genetics where the search for direct links between variability at the level of DNA and consequent changes in expression of phenotypic characters has long been a high priority.

However, there have been many other studies that have employed indirect techniques to assess genetic damage (for reviews see Møller and Mousseau 2006, 2015) and, when taken collectively, there is little doubt that the radioactive contaminants associated with the Chernobyl disaster have generated genetic damage and increased mutation rates, with many studies also finding phenotypic effects that were correlated to the levels of genetic damage reported. Surprisingly, the first summary of genetic effects stemming from exposure to Chernobyl-derived radiation was presented in Møller and Mousseau (2006: 205, Table 1), which listed 33 studies that had investigated mutations or cytogenetic effects of increased radiation around Chernobyl compared with control areas in a variety of plant and animal species. Although there was considerable heterogeneity in the results, 25 of the studies showed a significant increase in mutations or cytogenetic abnormalities related to radiation exposure. Several studies showed an increase in mutation rates for some loci, but not for others. However, many studies were based on small sample sizes, with a resulting low statistical power and were thus unable to show differences 
of 25 per cent as being statistically significant. Only four of these studies investigated germ-line mutations (i.e. mutations that could be passed to the next generation) and these all found significant increases. Of relevance here is the fact that many of these studies were not even considered by the highly influential International Atomic Energy Agency (IAEA) Chernobyl Forum reports, which downplayed the potential injury to natural populations.

Møller and Mousseau (2015) have recently extended their studies of mutation rates in Chernobyl populations and used a meta-analysis to examine the effects of radiation in Chernobyl across 45 published studies, covering 30 species. Meta-analysis is a relatively new statistical technique that permits the combination of datasets from disparate sources to permit global analyses of hypotheses of interest. Based on their meta-analysis, the overall effect size of radiation effects, estimated as Pearson's productmoment correlation coefficient, was very large $(E=0.67$; 95 per cent confidence intervals (CI) 0.59 to 0.73 ), accounting for 44.3 per cent of the total variance in an unstructured random-effects model (Møller and Mousseau 2015: 2, Figure 1). In simple terms, this means that radiation effects explained almost half of the total variation observed among studies, which is extraordinary by any standard. By using a 'fail-safe' sensitivity analysis, it was possible to determine just how robust this finding was. Fail-safe calculations reflect the number of unpublished null results that would be needed to eliminate this average effect size. In this study, the fail-safe number was 4135 demonstrating the extreme robustness of this finding (Rosenberg's method: 4135 at $p=0.05$ ). Indirect tests did not provide any evidence of publication bias. The effect of radiation on mutations varied among taxa, with plants showing a larger effect than animals. Humans were shown to have intermediate sensitivity of mutations to radiation compared to other species. Effect size did not decrease over time, providing no evidence for an improvement in environmental conditions. The surprisingly high mean effect size suggests a strong impact of radioactive contamination on individual fitness in current and future generations, with potentially significant populationlevel consequences, even beyond the area contaminated with radioactive material. Overall, this study provides perhaps the strongest evidence so far of the mutagenic consequences of chronic exposure to ionising radiation in natural populations. 
To date, there have been relatively few studies of genetic effects related to the Fukushima disaster. Joji Otaki of the University of the Ryukus in Okinawa, Japan, has conducted a series of seminal studies of butterflies exposed to radioactive contaminants associated with the Fukushima disaster and found strong evidence for increased mutation rates as a direct consequence of exposure to radionuclides (reviewed in Mousseau and Møller 2014). These studies were greatly strengthened by laboratory experiments that used both internal and external radiation sources, and these unambiguously validated observations of the elevated mutation rates and phenotypic effects observed in the field (Mousseau and Møller 2014). Later studies by Otaki's group provided additional support for acute and chronic effects of radiation effects, with effects decreasing over time, possibly due to reduced dose rates after several years. Of particular note was the suggestion that acquired mutations were in some cases transmitted to offspring. Collectively, these studies of butterflies provide some of the most rigorous and comprehensive experimental analyses of chronic radiation effects in natural populations.

Additional support for the hypothesis that low-dose rate exposures can lead to elevated mutation rates comes from a recent meta-analysis of the effects of naturally occurring radioactive materials on plant and animal populations around the world (Møller and Mousseau 2013). Natural radiation levels vary greatly across the planet largely in relation to variation in surface deposits of radioactive uranium and thorium. Well-known areas include Ramsar, Iran, Kerala, India, and Guarapara, Brazil, among many others. This study surveyed the results from more than 5,000 publications to arrive at 46 studies conducted with sufficient rigour to be included in the meta-analysis. Although many of the individual effects were small and statistically insignificant on their own, overall there were many more that were greater than zero than expected by chance, with an overall average effect size of 0.093 (95 per cent $\mathrm{CI}=0.039-0.171)$ indicating that exposure to naturally occurring radiation accounted for about 1 per cent of the variance in the traits examined. Albeit a small effect, this could still prove significant on an evolutionary timescale. The principal conclusion from this analysis was that there is extensive evidence for small, but significant negative effects of natural variation in background radiation on immune systems, mutation rates, and disease expression across a range of different animals and plants (Møller and Mousseau 2013). In other words, there was no evidence of any threshold below which effects are not potentially observable given sufficient statistical power. Studies of 
naturally radioactive areas may also provide opportunities to investigate evolutionary processes of adaptation, although to our knowledge no such studies have yet been conducted. Of more relevance here, perhaps, is the finding that 'natural background levels' of radiation can be sufficient to cause injury to individuals, contrary to frequent statements by nuclear industry and regulatory bodies that, because emissions from nuclear power plants are often of the same order of magnitude as background levels, they do not need to be considered from a public health perspective. This study suggests that there are good reasons to consider radioactive releases, even if they are similar to natural background levels.

\section{Developmental effects: Albinism, asymmetry, brain size, cataracts, sperm, and tumors}

There is an increasing array of empirical studies in Chernobyl, and now Fukushima, that document a wide range of physiological, developmental, morphological, and behavioural consequences of exposure to radioactive contaminants. It is presumed that most of these effects have an underlying genetic basis, although in some cases direct toxicity cannot be ruled out. Among the first visible signs of exposure were the appearance of white spots on feathers of birds and perhaps the fur of mammals (i.e. cattle in Fukushima). These 'partial albinos' (also sometimes referred to as partial leucism) have been well-documented for barn swallows in Chernobyl and for a number of other bird species as well (Mousseau and Møller 2014). Barn swallows with aberrant white feathers were first detected in Fukushima in 2012 by amateur bird watchers and were observed in apparently increasing frequencies in 2013 . However, such a trend could in part be related to a 'screening effect' due to higher levels of scrutiny for this trait following the disaster and further investigation is needed. Although such partial albinos are believed to have reduced probabilities of survival, there are sufficient data to suggest that this character can be inherited and may at least in part result from a mutation(s) in the germ line, based on parent-offspring resemblance. Although the presence of white feathers in and of itself seems unlikely to directly affect individual performance (i.e. reproduction and survival), it may serve as a useful biomarker for radiation effects on individuals. Further research is needed to determine any links between the expression of this trait and any underlying genetic or physiological mechanism related to radiation exposure. 
Analysis of gametes has served as a proxy for estimates of germ line mutation rates for several species of birds in Chernobyl. For example, it has been reported that the frequency of abnormal sperm in barn swallows was up to 10 times higher for Chernobyl birds as compared to sperm from males living in control areas (Mousseau and Møller 2014). It was found that abnormality rates were correlated with reduced levels of antioxidants in the blood, liver, and eggs of these birds, supporting the hypothesis that antioxidants likely play a significant role in protecting DNA from the direct and indirect consequences of exposure to radionuclides. And a more recent analysis of Chernobyl birds found that in nine out of 10 species examined, sperm abnormality rates were much larger for birds living in Chernobyl than those living in control areas across Europe, with the highest damage levels observed for species with longer sperm, suggesting that sperm abnormalities are likely common for birds living in radioactive areas. Similar effects on sperm morphology of small rodents have recently been reported (Kivisaari et al. 2016). It has been found that barn swallow sperm swimming ability is negatively related to radiation levels and that plasma oxidative status could predict sperm performance, further supporting the role antioxidants are known to play in protecting spermatogenesis from the effects of ionising radiation (reviewed in Mousseau and Møller 2014). Overall, these studies provide convincing evidence that spermatogenesis can be significantly impacted by low-dose radiation and the resulting male infertility may in part explain the smaller population sizes of many species that have been documented for the region (see below).

Studies of plant pollen and seed germination may also be informative with respect to radiation effects on reproductive tissue and hence fitness of individuals. A recent study of 111 plant species in Chernobyl found small but significant negative effects of radiation on pollen viability (Møller, Shyu, and Mousseau 2016), which may in part explain reduced germination rates in many of these species (Møller and Mousseau 2017).

Many other cell types and tissues have been shown to be affected by Chernobyl contaminants. For example, it has been demonstrated that the frequency of visible tumors on birds was significantly higher in radioactive areas, presumably reflecting elevated mutation rates in somatic tissues (reviewed in Mousseau and Møller 2014). Visible tumor rates in birds from Chernobyl were in excess of 15 per 1,000 birds, while tumors have never been reported for Danish populations despite extensive surveys 
(0 per 35,000 birds observed). Recent surveys of rodents from Chernobyl suggest increased frequency of tumors related to radiation dose as measured by whole body burdens of cesium-137.

Radiation cataract was detected in the eyes of atomic bomb survivors shortly after the end of the Second World War and showed a very significant dose response relationship (Otake and Schull 1991). Similarly, in Chernobyl, the frequency and magnitude of cataract expression in eyes was related to radiation exposure: birds from areas with high background radiation were more likely to display opacities in one or both eyes (Mousseau and Møller 2014). As with radiation-related cataract in humans, there was no relationship with the age of the birds, further supporting the hypothesis that radiation was the underlying cause of cataract expression. Lehmann et al. (2016) recently reported significantly increased rates of cataracts in rodents living in radioactively contaminated regions of Ukraine providing additional support for the use of cataract incidence as a reliable biomarker for exposure to ionising radiation. Although never explicitly tested, it seems likely that impaired vision related to cataracts would have significant fitness consequences for animals (for example, the probability of evading predators or finding food).

Neurological development has long been known to be sensitive to the effects of ionising radiation. Many studies of prenatally exposed survivors of the atomic bombings of Hiroshima and Nagasaki suggested that serious mental retardation and small head sizes are a direct consequence of exposure to ionising radiation (Otake and Schull 1998). Chernobyl birds also show reduced brain size in regions of high radioactivity and smaller brain size was associated with reduced survival prospects (reviewed in Mousseau and Møller 2014). Similar effects have also been observed for rodents living in both Chernobyl and Fukushima (Mappes et al. 2016).

A wide range of other morphological and behavioural abnormalities have been reported for wild organisms living in radioactive regions of Chernobyl. Time, effort, and imagination appear to be the only constraints to the discovery of the biological consequences of the Chernobyl accident. This is exemplified by a recent study of calling song behaviour in the European cuckoo, Cuculus canorus (Møller et al. 2016). In this study, the authors documented the number of 'syllables' in the calls of 129 male cuckoos as well as the occurrence of 'aberrant' calls from a number of locations in Ukraine spanning a large range of radioactivity, from 0.01 to 218 microSievert per hour $(\mu \mathrm{Sv} / \mathrm{h})$. Overall, males produced fewer 
and more aberrant syllables in radioactively contaminated areas, and this effect persisted even after correcting for the potential effects of other environmental variables. Although it is not possible to extrapolate from the call of a cuckoo to health effects in humans, radioactive contaminants can very clearly influence natural systems in a wide variety of ways.

\section{Population abundances and biodiversity in regions of high radiation}

A key issue for conservation biologists concerns the fitness consequences of mutation accumulation and resulting developmental effects that have been observed for wild populations living in Chernobyl and Fukushima. To this end, we have conducted demographic studies aimed at documenting population sizes, numbers of species (i.e. biodiversity), sex ratios, survival and reproductive rates, and patterns of immigration for animals in both Chernobyl and Fukushima. Because of the highly heterogeneous nature of radionuclide deposition inside the contaminated regions of Chernobyl and Fukushima, it is possible to identify areas that represent the full spectrum of radiation levels, from relatively 'clean' uncontaminated habitats all the way to large areas of very high radiation levels, all within short geographical distances. This heterogeneity makes it possible to conduct highly replicated tests for the effects of radiation on biological populations and communities for a single large-scale event. In effect, the distribution of radioactive contaminants, especially in Chernobyl, is more akin to a mosaic or quiltwork than diffusion from a point, allowing the uncoupling of radiation levels from distance from the source. It is this lack of geographic structure for radiation levels when combined with multiple tests for radiation effects across multiple habitat types that permits a sensitive analysis of radiation effects independent of other biotic and abiotic factors.

\section{Abundance and diversity of birds, butterflies, and other invertebrates}

Comprehensive surveys of animal abundance and diversity in Chernobyl were conducted by Møller and Mousseau starting in the mid-2000s. The basic sampling protocol was a 'massively replicated biotic inventory' design whereby point counts of birds and invertebrates (chiefly, butterflies, 
dragonflies, bees, grasshoppers, and spiders) were conducted at about 300 locations across northern Ukraine and southeastern Belarus in 2006-08. An identical protocol was used to conduct surveys in Fukushima at 400 distinct locations in 2011-16. To date (2016), a total of 1,146 and 1,900 biotic inventories have been generated for the Chernobyl and Fukushima regions, respectively. In addition to quantitative estimates of animal abundances and species diversity at each site, a large number of additional biotic and abiotic factors were measured or estimated, including the type of vegetation, the distance to open water, soil type, ambient meteorological conditions, latitude, longitude, elevation, and time of day. All of these variables were included in a multivariate model and used to generate predictions for expected numbers of organisms of each species or group for each location. This model was then used to provide estimates of the variation in numbers explained by radiation independent of all the other potentially contributing factors; in essence, a partial relationship between abundance and radiation. To our knowledge, this approach has not been used in this way before to assess radioactively contaminated areas, although it has been used for monitoring bird populations in Europe and North America since the 1960s. This approach is perhaps the only solution for complex ecological questions of this type, short of large-scale experimental manipulations, which are generally not possible for testing the effects of nuclear fission products at a landscape scale. This approach has the added advantage of permitting assessment of ecological effects even in the absence of pre-disaster baseline data, as it uses contemporary observations of distribution and abundance from unaffected areas to infer expected patterns in contaminated areas.

Contrary to popular notions, in 2006-09 the abundance and diversity of forest and grassland birds in Chernobyl were dramatically lower in contaminated areas, showing a dose-response-like relationship, with about one-third as many birds and half as many species present in high contamination areas relative to that predicted by the models and abundances found in relatively 'clean' parts of the same general region (reviewed in Mousseau and Møller 2014). Although not every species showed declines with radiation levels, and a few even appeared to be unaffected (Galván et al. 2014) and perhaps showed evolutionary adaptation to radiation, the overall patterns of decline were very apparent and the analyses were statistically robust. Birds of prey also showed patterns of reduced numbers in contaminated regions of Chernobyl, although it was not apparent if reduced numbers were a consequence of direct exposure to radionuclides 
via ingestion or via indirect effects on behaviour mediated by reduced prey. In addition to population censuses, there are other lines of evidence supporting the observed decline in population sizes of birds in Chernobyl including changes in adult sex ratios (more males than females), and reductions by half in the number of older birds relative to juveniles and one-year-olds. Also, there was evidence from analyses of stable isotopes in feathers that the Chernobyl region is acting like a population sink with a higher proportion of immigrants present than in control areas or when compared to birds in historical museum collections from the same area.

The overall pattern was very similar for birds in Fukushima in July 2011, with the strength of the negative relationship between abundance and radiation significantly stronger in Fukushima when comparing the 14 bird species that were common to both regions (Mousseau and Møller 2014). The observed stronger relationship in Fukushima could reflect the difference between acute and chronic exposures, with Chernobyl bird populations showing a response to $20+$ years of selection for resistance, or this could reflect the effects of other radionuclides (for example, iodine-131 and cesium-134) that were present at high levels in Fukushima during the spring of 2011 that are no longer present in Chernobyl.

Field studies in Fukushima were also conducted in 2011-14, and the initial analyses showed a strengthening of the negative relationship between ambient radiation levels and abundance and species richness at a given site over time (Møller et al. 2015; Møller, Nishiumi, and Mousseau 2015). Although no comprehensive surveys of raptors in Japan have yet been conducted, a recent study of goshawk (Accipiter gentilis Fujiyama) has reported significant declines in reproduction for this bird of prey in Fukushima following the disaster (Murase et al. 2015), although only three study areas were included in the analysis and thus attribution of the observed effect to radionuclide exposure is preliminary. Surveys of barn swallows showed significant drop-offs in abundance in the more radioactive regions of Fukushima, although preliminary analyses did not indicate any relationship with genetic damage to blood cells in nestlings (Bonisoli-Alquati et al. 2015).

In the first study of its sort, Garnier-Laplace et al. (2015) calculated doses for 57 species of birds (almost 7,000 individuals) living in Fukushima following the nuclear disaster of 11 March 2011 that were surveyed by Møller et al. (2015), and Møller, Nishiumi, and Mousseau (2015). Doses were calculated based on radiological conditions at the point 
of observation and corrected by including ecological and life history attributes of the species in the model. Dose was used to predict total number of birds while statistically controlling for potentially confounding environmental variables (for example, habitat type, elevation, presence of water bodies, ambient meteorological conditions, and time of day). Total dose was found to be a strong predictor of abundances $(P<0.0001)$, which showed a proportional decline with increasing doses with no indications of a threshold or intermediate optimum. Overall, the $\mathrm{ED}_{50 \%}$ (the total absorbed dose causing a 50 per cent reduction in the total number of birds) was estimated to only be 0.55 gray.

It is interesting to note that, as a group, butterflies also showed significant declines with radiation levels in both Chernobyl and Fukushima (Mousseau and Møller 2014). We speculate that there is something peculiar about the female ZW sex determination system shared by birds and butterflies (i.e. females are heterogametic) that make these groups particularly vulnerable to mutagenic substances.

Unlike mammals where males are 'XY' and females are 'XX' with respect to sex chromosomes, in both birds and butterflies it is the female that is the equivalent of XY. This 'reversal' of the genetic system underlying sex determinism could greatly enhance the deleterious effects of mutations on reproduction and hence population growth rates.

Our hypothesis is that because in these groups the sex that is responsible for egg production is heterogametic, mutational load effects on reproduction stemming from mutation accumulation on the $\mathrm{Z}$ chromosome are likely to be expressed immediately following exposure as opposed to species where females are homogametic, as is the case for most sexually reproducing organisms (for example, mammals). In species where the female is homogametic (i.e. XX in mammals), deleterious effects of mutations on these chromosomes may not be expressed immediately because of redundancies in the genetic material. This might be particularly important given the apparent lack of gene dosage compensation in birds and Lepidoptera. In addition, slightly deleterious mutations may accumulate faster on sex chromosomes than on autosomes, and this could be, at least in part, responsible for the observed greater sensitivity to radiation reported for birds and Lepidoptera. 
Put more simply, female birds and butterflies may be more likely to express accumulated mutations arising from ionising radiation than females from other species because of their reversed sex determination system. And, because females are in large part responsible for propagation of the species (i.e. they make the eggs), mutational effects could lead to direct effects on fecundity, which is often the most important determinant of population growth rates.

In most other invertebrate groups examined (for example, grasshoppers, dragonflies, bees, and spiders), population sizes were significantly reduced in areas of high contamination in Chernobyl 20+ years after the disaster, while there was no evidence for similar declines in Fukushima; in fact, spiders showed significant increases in numbers, at least during the first summer following the disaster (Mousseau and Møller 2014). It has been proposed that such differences in the time course for population effects might reflect the consequences of multi-generational mutation accumulation of recessive deleterious mutations in Chernobyl, which is also consistent with the immediate effects on birds and butterflies observed in Fukushima. Alternatively, increases in spider numbers could simply reflect a reduction in predation pressure (for example, birds), a finding similar to that reported for large mammals living in the Chernobyl zone where the lack of hunting pressure has been associated with increased population sizes in some species.

Recent evidence suggests that DNA repair may also be involved in determining sensitivity to the mutagenic properties of radionuclides. A recent analysis found a significant relationship between the strength of population declines of a given species with radiation and historical mitochrondial DNA substitution rates for 32 species of birds in Chernobyl (Møller et al. 2010). Species with higher substitution rates showed the greatest declines with radiation levels, suggesting that variation in DNA repair capability may be influencing population success, although this hypothesis remains to be tested experimentally. In essence, individuals of some species are less able to cope with the increased levels of genetic damage associated with ionising radiation in Chernobyl and Fukushima. 


\section{Large mammals: A special case?}

Recently, it has been suggested that some of the large mammals of the Chernobyl Exclusion Zone are thriving, and perhaps it would not be surprising if this were indeed the case for animals that normally face significant hunting pressure. Inside the exclusion zones of both Chernobyl and Fukushima, hunting pressures are significantly reduced, if not completely eliminated, thus providing a refuge for game animals. Two recent studies have suggested increased numbers of wolves, deer, elk, and wild boar living in the Chernobyl Exclusion Zone. However, these studies were conducted in a manner that did not permit rigorous analysis of radiation effects on relative abundances or animal health. Prior, finer-scaled analyses of mammal distribution and abundance have demonstrated significantly fewer individuals of all species (except wolves) in the more radioactive areas of the zone (Mousseau and Møller 2014), and more recent studies of rodents have demonstrated significant decreases in abundances and fertility (Mappes et al. 2016), as well as high frequencies of cataracts in the more radioactive areas of the Chernobyl Exclusion Zone (Lehmann et al. 2016). In sum, these studies of mammals suggest that, although there are clear indications of radiation effects, hunting pressure must also be factored into such analyses. Similar effects have recently been reported for fish species in Fukushima that would normally have been under significant fishing pressure.

\section{Adaptation to radiation?}

Evolution by natural selection is an inevitable and ubiquitous consequence of simple biological processes: all organisms are capable of reproduction, some more, some less, with some portion of the variation in reproductive success being related to the phenotypic attributes of the individual that are more or less genetically determined. Previous studies of genetic variability within natural populations (for example, Mousseau and Roff 1987) suggest that genetic variation generally exists in most species for most characters, and laboratory studies have repeatedly demonstrated that some organisms can adapt to high radiation levels (for example, tardigrades; Jönsson et al. 2008). However, relatively few studies have attempted to assess adaptive responses to radiation of organisms living under natural conditions. Møller and Mousseau (2016) recently reviewed all purported studies of evolutionary responses of organisms living in Chernobyl and found very 
little evidence to suggest that adaptation might be a common response to ionising radiation. Of the 14 studies conducted with sufficient rigour to address this question, only one study (of bacteria) showed any signs of an evolved, adaptive response (Ruiz-González et al. 2016). In addition, there are no studies to date that provide any evidence for hormesis in these natural populations. There are several reasons why adaptation might not evolve under radioactive conditions, including the possibility that there is no genetic variation within a population for adaptive responses, or that there may have been insufficient time since the accident to allow for evolutionary responses. Studies of naturally radioactive regions of the world where organisms have had millennia to respond to this type of selection pressure may provide valuable insights to the mechanisms underlying adaptive responses as well as likelihood of such responses in regions affected by nuclear accidents (Mousseau and Møller 2014).

\section{Ecosystem consequences of nuclear accidents}

Ecosystems provide many of the basic requirements for life on the planet. Ecosystem services related to humans include the provisioning of water, medicines, foods, and plant productivity, among many other functions. Given the wide range of radiation effects on individuals, populations, and communities of plants, animals, and microbes, it would not be surprising to find consequences at the level of ecosystem function. To date, very few ecosystem-level studies have been performed at either Chernobyl or Fukushima. However, based on limited data, it is very clear that ecosystems are not immune to the impacts of nuclear accidents. Recent studies have found that plant primary productivity is negatively impacted by radiation stemming from the Chernobyl accident, as evidenced by reduced growth rates of trees following 1986, especially during years when there was additional stress induced by drought (Mousseau et al. 2013). Experimental studies of the rate of decomposition of leaf litter at the soil surface have demonstrated dramatically reduced decomposition rates in areas of high ambient radiation that likely reflect effects on the microbial community (especially fungi and bacteria) (Mousseau et al. 2014). It is very likely that such effects on decomposition have large cascading effects on nutrient turnover rates in the soil, potentially impacting plant productivity at landscape scales. In fact, the reduced growth rates of trees observed in Chernobyl could be in part related 
to the indirect consequences of radiation effects on the soil microbial communities, which are often essential for the acquisition of mineral nutrients by plants. Other studies have documented radiation effects on the interactions between plants, insect pollinators, and fruit productivity that are clearly affecting other components of the ecosystem in negative ways (for example, Møller, Barnier, and Mousseau 2012). And it now seems apparent that stress due to radiation can interact with climate change to influence patterns of ecological succession in ways that may pose an ongoing threat to human populations adjacent to contaminated regions. For example, reduction in decomposition rates in radioactive areas has resulted in the accumulation of dead organic matter (for example, leaf litter) at the soil interface, thus dramatically increasing the fuel available for forest fires, which have increased in frequency and intensity in recent years as a consequence of climate change in the region (Mousseau et al. 2014). Given that this soil litter is itself often highly radioactive, forest fires have the potential to volatilise radionuclides and to disperse these contaminants to populated regions in surrounding countries (Evangeliou et al. 2015), as was demonstrated following several fires in the Chernobyl Exclusion Zone during the summer of 2015 (Evangeliou et al. 2016).

\section{Concluding remarks}

In conclusion, the radiological disasters at Chernobyl and Fukushima provide a unique opportunity to investigate genetic, ecological, evolutionary, and ecosystem consequences of acute and chronic exposures to mutagenic sources in natural populations at regional and landscape scales. Recent advances suggest many small and large effects on biological systems, from molecules to ecosystems, that will likely influence ecosystem form and function for decades to centuries to come. Recent surveys of population effects in Chernobyl (Garnier-Laplace et al. 2013) suggest that populations living under the full range of natural stressors (biotic and abiotic) are almost 10 times more sensitive to ionising radiation than predicted by conventional approaches used by some regulatory and governmental agencies, providing some potential insights to the cause of the apparent discrepancy between empirical ecological studies and predictions from conventional radio-ecological models of radiation effects. The opportunity to compare and contrast organisms from both Chernobyl and Fukushima provides for a possible level of scientific rigour (i.e. replication) not previously available for studies of this sort, as well 
as analysis of the time frame over which responses may occur, and the development of predictive models to aid the management and conservation of biological systems following future nuclear accidents. Given recent advances in molecular genetic technologies, it seems likely that much new knowledge could be gained from a sustained and expansive investment in basic research related to the biological effects of radioactive mutagens within an ecosystem context that could extend far beyond the disasters at Fukushima and Chernobyl. Given the near certainty of additional nuclear accidents small and large in the near future, investment in the basic research needed to characterise the environmental consequences of past accidents would seem prudent.

\section{Acknowledgements}

We thank our many friends and colleagues in Ukraine, Belarus, Japan, and elsewhere, without whom this work would never have been conducted. Our studies have been financially supported by a large number of sources including the Centre National de la Recherche Scientifique (CNRS, France), the National Science Foundation, the National Institutes of Health, CRDF Global, the National Geographic Society, the University of South Carolina, the Fulbright Foundation, the Samuel Freeman Charitable Trust, the American Council of Learned Societies, Chubu University, and private citizens of the US and Japan. This essay is dedicated to the memory of Eugene Pisanets, Dmitry Grodzinsky, and Alexey Yablokov.

\section{References}

Bonisoli-Alquati, A., K. Koyama, D. J. Tedeschi, W. Kitamura, H. Suzuki, S. Ostermiller, E. Arai, A. P. Møller, and T. A. Mousseau, 2015. Abundance and genetic damage of barn swallows from Fukushima. Scientific Reports 5: 9432. doi.org/10.1038/srep09432

Burris, J. E., J. C. Bailar III, H. L. Beck, A. Bouville, P. S. Corso, P. J. Culligan, P. M. Deluca Jr, R. A. Guilmette, G. M. Hornberger, M. Karagas, R. Kasperson, J. E. Klaunig, T. Mousseau, S. B. Murphy, R. E. Shore, D. O. Stram, M. Tirmarche, L. Waller, G. E. Woloschak, and J. J. Wong, 2012. Analysis of Cancer Risks in Populations Near Nuclear Facilities: Phase I. Washington, DC: National Academies Press. 
Evangeliou N., Y. Balkanski, A. Cozic, W. M. Hao, F. Mouillot, K. Thonicke, R. Paugam, S. Zibtsev, T. A. Mousseau, R. Wang, B. Poulter, A. Petkov, C. Yue, P. Cadule, B. Koffi, J. W. Kaiser, and A. P. Møller, 2015. Fire evolution in the radioactive forests of Ukraine and Belarus: Future risks for the population and the environment. Ecological Monographs 85(1): 49-72. doi.org/10.1890/14-1227.1

Evangeliou, N., S. Zibtsev, V. Myroniuk, M. Zhurba, T. Hamburger, A. Stohl, Y. Balkanski, R. Paugam, T. A. Mousseau, A. P. Møller, and S. I. Kireev, 2016. Resuspension and atmospheric transport of radionuclides due to wildfires near the Chernobyl nuclear power plant (CNPP) in 2015: An impact assessment. Scientific Reports 6: 26062. doi.org/10.1038/srep26062

Fairlie, I., 2014. A hypothesis to explain childhood cancers near nuclear power plants. Journal of Environmental Radioactivity 133: 10-17. doi. org/10.1016/j.jenvrad.2013.07.024

Galván, I., A. Bonisoli-Alquati, S. Jenkinson, G. Ghanem, K. Wakamatsu, T. A. Mousseau, and A. P. Møller, 2014. Chronic exposure to lowdose radiation at Chernobyl favours adaptation to oxidative stress in birds. Functional Ecology 28(6): 1387-403. doi.org/10.1111/13652435.12283

Garnier-Laplace, J., K. Beaugelin-Seiller, C. Della-Vedova, J. M. Métivier, C. Ritz, T. A. Mousseau, and A. P. Møller, 2015. Radiological dose reconstruction for birds reconciles outcomes of Fukushima with knowledge of dose-effect relationships. Scientific Reports 5: 16594. doi. org/10.1038/srep16594

Garnier-Laplace, J., S. Geras'kin, C. Della-Vedova, K. Beaugelin-Seiller, T. G. Hinton, A. Real, and A. Oudalova, 2013. Are radiosensitivity data derived from natural field conditions consistent with data from controlled exposures? A case study of Chernobyl wildlife chronically exposed to low dose rates. Journal of Environmental Radioactivity 121: 12-21. doi.org/10.1016/j.jenvrad.2012.01.013

Jönsson, K. I., E. Rabbow, R. O. Schill, M. Harms-Ringdahl, and P. Rettberg, 2008. Tardigrades survive exposure to space in low Earth orbit. Current Biology 18(17): R729-31. doi.org/10.1016/j. cub.2008.06.048 
Kivisaari, K., Z. Boratynski, S. Calhim, P. Lehmann, T. Mappes, T. A. Mousseau, and A. P. Møller, 2016. Cut to the chase: Radiation effects on sperm structure at Chernobyl. In review.

Lehmann, P., Z. Boratynski, T. Mappes, T. A. Mousseau, and A. P. Møller, 2016. Fitness costs of increased cataract frequency and cumulative radiation dose in natural mammalian populations from Chernobyl. Scientific Reports 6: 19974. doi.org/10.1038/srep19974

Mappes, T., Z. Boratynski, K. Kivisaari, G. Milinevski, T. A. Mousseau, A. P. Møller, E. Tukalenko, and P. Watts, 2016. Radiation effects on breeding and population sensitivity in a key forest mammal of Chernobyl. In review.

Møller, A. P., and T. A. Mousseau, 2006. Biological consequences of Chernobyl: 20 years on. Trends in Ecology and Evolution 21(4): 200-7. doi.org/10.1016/j.tree.2006.01.008

Møller, A. P., and T. A. Mousseau, 2013. The effects of natural variation in background radioactivity on humans, animals and other organisms. Biological Reviews of the Cambridge Philosophical Society 88(1): 22654. doi.org/10.1111/j.1469-185X.2012.00249.x

Møller, A. P., and T. A. Mousseau, 2015. Strong effects of ionizing radiation from Chernobyl on mutation rates. Scientific Reports 5: 8363. doi.org/10.1038/srep08363

Møller, A. P., and T. A. Mousseau, 2016. Are animals and plants adapting to low-dose radiation at Chernobyl? Trends in Ecology and Evolution 31(4): 281-9. doi.org/10.1016/j.tree.2016.01.005

Møller, A. P., and T. A. Mousseau, 2017. Radiation levels affect pollen viability and germination among sites and species at Chernobyl. International Journal of Plant Species 178(7): 537-45. doi. org/10.1086/692763

Møller, A. P., F. Barnier, and T. A. Mousseau, 2012. Ecosystem effects 25 years after Chernobyl: Pollinators, fruit set, and recruitment. Oecologia 170: 1155-65. doi.org/10.1007/s00442-012-2374-0 
Møller A. P., J. Erritzøe, F. Karadas, and T. A. Mousseau, 2010. Historical mutation rates predict susceptibility to radiation in Chernobyl birds. Journal of Evolutionary Biology 23(10): 2132-42. doi.org/10.1111/ j.1420-9101.2010.02074.x

Møller, A. P., F. Morelli, T. A. Mousseau, and P. Tryjanowski, 2016. The number of syllables in Chernobyl cuckoo calls reliably indicate habitat, soil and radiation levels. Ecological Indicators 66: 592-7. doi. org/10.1016/j.ecolind.2016.02.037

Møller, A. P., T. A. Mousseau, I. Nishiumi, and K. Ueda, 2015. Ecological differences in response of bird species to radioactivity from Chernobyl and Fukushima. Journal of Ornithology 156(S1): 287-96. doi. org/10.1007/s10336-015-1173-x

Møller, A. P., I. Nishiumi, and T. A. Mousseau, 2015. Cumulative effects of radioactivity from Fukushima on the abundance and biodiversity of birds. Journal of Ornithology 156(S1): 297-305. doi.org/10.1007/ s10336-015-1197-2

Møller, A. P., J. C. Shyu, and T. A. Mousseau, 2016. Ionizing radiation from Chernobyl and the fraction of viable pollen. International Journal of Plant Sciences 177(9): 727-35. doi.org/10.1086/688873

Mousseau, T. A., G. Milinevsky, J. Kenney-Hunt, and A. P. Møller, 2014. Highly reduced mass loss rates and increased litter layer in radioactively contaminated areas. Oecologia 175(1): 429-37. doi.org/10.1007/ s00442-014-2908-8

Mousseau, T. A., and A. P. Møller, 2014. Genetic and ecological studies of animals in Chernobyl and Fukushima. Journal of Heredity 105(5): 704-9. doi.org/10.1093/jhered/esu040

Mousseau, T. A., and D. A. Roff, 1987. Natural selection and the heritability of fitness components. Heredity 59(Pt 2): 181-97. doi. org/10.1038/hdy.1987.113

Mousseau, T. A., S. M. Welch, I. Chizhevsky, O. Bondarenko, G. Milinevsky, D. Tedeschi, A. Bonisoli-Alquati, and A. P. Møller, 2013. Tree rings reveal extent of exposure to ionizing radiation in Scots pine Pinus sylvestris. Trees: Structure and Function 27(5): 1443-53. doi. org/10.1007/s00468-013-0891-z 
Murase, K., J. Murase, R. Horie, and K. Endo, 2015. Effects of the Fukushima Daiichi accident on goshawk reproduction. Scientific Reports 5: 9405. doi.org/10.1038/srep09405

Otake, M., and W. J. Schull, 1991. A review of forty-five years study of Hiroshima and Nagasaki atomic bomb survivors: Radiation cataract. Journal of Radiation Research 32: 283-93. doi.org/10.1269/jrr.32. SUPPLEMENT_283

Otake, M., and W. J. Schull, 1998. Radiation-related brain damage and growth retardation among the prenatally exposed atomic bomb survivors. International Journal of Radiation Biology 74(2): 159-71. doi.org/10.1080/095530098141555

Ruiz-González, M. X., G. Á. Czirják, P. Genevaux, A. P. Møller, T. A. Mousseau, and P. Heeb, 2016. Resistance of feather-associated bacteria to intermediate levels of ionizing radiation near Chernobyl. Scientific Reports 6: 22969. doi.org/10.1038/srep22969

Samet, J. M., and J. Seo, 2016. The financial costs of the Chernobyl nuclear power plant disaster: A review of the literature. Zurich: Green Cross Switzerland.

US GAO (Government Accountability Office), 2011. Nuclear Regulatory Commission: Oversight of underground piping systems commensurate with risk, but proactive measures could help address future leaks. Report GAO-11-563. Washington, DC: US Government Accountability Office.

Wheatley, S., B. K. Sovacool, and D. Sornette, 2016. Reassessing the safety of nuclear power. Energy Research \& Social Science 15: 96-100. doi.org/10.1016/j.erss.2015.12.026 
This text is taken from Learning from Fukushima: Nuclear power in East Asia, edited by Peter Van Ness and Mel Gurtov, published 2017 by ANU Press, The Australian National University, Canberra, Australia.

dx.doi.org/10.22459/LF.09.2017.09 